\title{
Logic control of microfluidics with smart colloid $\dagger$
}

\author{
Limu Wang, ${ }^{a}$ Mengying Zhang, ${ }^{a}$ Jiaxing Li, ${ }^{b}$ Xiuqing Gong ${ }^{a}$ and Weijia Wen ${ }^{* a b}$
}

\author{
Received 6th May 2010, Accepted 24th August 2010 \\ DOI: 10.1039/c0lc00003e
}

\begin{abstract}
We report the successful realization of a microfluidic chip with switching and corresponding inverting functionalities. The chips are identical logic control components incorporating a type of smart colloid, giant electrorheological fluid (GERF), which possesses reversible characteristics via a liquid-solid phase transition under external electric field. Two pairs of electrodes embedded on the sides of two microfluidic channels serve as signal input and output, respectively. One, located in the GERF microchannel is used to control the flow status of GERF, while another one in the ither micro-fluidic channel is used to detect the signal generated with a passing-by droplet (defined as a signal droplet). Switching of the GERF from the suspended state (off-state) to the flowing state (on-state) or vice versa in the micro-channel is controlled by the appearance of signal droplets whenever they pass through the detection electrode. The output on-off signals can be easily demonstrated, clearly matching with GERF flow status. Our results show that such a logic switch is also a logic IF gate, while its inverter functions as a NOT gate.
\end{abstract}

\section{Introduction}

Discussions of digital microfluidics (DMF) are usually confined to the context of electrowetting-on-dielectric (EWOD) fluid control systems, ${ }^{1,2}$ which are thought to be the most promising technique to realize digital microfluidics, ${ }^{3}$ whereas the logic operation is actually conducted by a peripheral computer system, and droplets respond passively to control signals. With EWOD systems, the paradigm for pure fluid/droplet logic, in which the fluid responds only to droplet (fluid) inputs, has somehow been neglected. Several precedent works have been done in pure fluidic logic, for example, geometry decided bubble logic ${ }^{4}$ and continuous phase logic. ${ }^{5}$ However, the above techniques are usually based on pressure resistance, which results in a specific designed channel configuration for each logic operation, and thus the inevitable amplified perturbation in fluidic system usually occurs. Another trend of microfluid logic systems is the use of pressure controlled valves. ${ }^{6}$ One difficulty there, however, is the subsequent fluid control, which is often manipulated by a gas/fluid valve. The pressure gain of fluid signals, or pressure difference between variable fluid signals, is utilized to control those valves. The specific problem is that the pressure gain is usually less than unity, which is not an ideal choice for further process control. A solution suggested is a kind of novel implanted valve controlled by a pressure gain greater than unity, applied in static digital microfluid control logic. ${ }^{7}$ Another difficulty in this trend, is that the control unit always calls for multilayer architectures: the sealing between different layers, the alignment among fine

${ }^{a}$ Nano Science and Nano Technology program, The Hong Kong University of Science and Technology, Clear Water Bay, Hong Kong. E-mail: phwen@ust.hk; Fax: +852 23581652;Tel: +852 23587979

${ }^{b}$ Physics Department and KAUST-HKUST Micro/Nanofluidic Joint Laboratory, The Hong Kong University of Science and Technology, Clear Water Bay, Hong Kong

$\dagger$ Published as part of a LOC themed issue dedicated to Chinese Research: Guest Editor Professor Bingcheng Lin. structures, and the fabrication techniques make the realization of total fluid logic systems a big challenge.

We are motivated by the concept of Lab on a Chip systems, ${ }^{\mathbf{8}, 9}$ where microfluid logic has a promising future in simplifying control schemes and reducing parallel equipment, by implanting an internal droplet signal detection and processing module. Recently, we have successfully realized on-chip fluidic control by constructing microvalves ${ }^{10}$ and micropumps ${ }^{11}$ for fluid logic, and we further introduced smart droplet control ${ }^{12}$ by active control components. These devices were purposely designed for fluid logic execution, storage, and display, ${ }^{13}$ and were driven by a giant electrorheological fluid (GERF), ${ }^{14}$ a type of smart colloid with tunable yield stress or "hardness" under an external electric field. ${ }^{15,16}$

Here we introduce a new type of pure fluid (droplet) logic control module, a microfluid switch and its inverter, which serve IF and NOT droplet logic functions, respectively. The chip is of a simple one-layer architecture, boasting a high response precision, and very short response time on the order of milliseconds. We use the differences of conductivity/dielectric characteristics between fluids to sense the droplet signal and utilize GERF as the working medium for signal response, subsequent detection and fluid control. ${ }^{12,13}$ The key to this logic design is its incorporation of GERF, an extraordinary smart fluidic material that can serve as a fluid-electric-mechanical interface and as a working medium for the carriage of input fluidic signals - in much the same sense as a current carries information in an electrical system. The output GERF signal can be accessed in droplet form, or can be converted to electrical or mechanical forma kind of signal which maintains three forms simultanously. The yield pressure gain imparted by the resultant GERF droplet for subsequent fluid control can be much larger than unity, offering easy manipulation, especially in electrical/digital manipulation of fluid. By employing a soft conductive composite (AgPDMS $)^{17}$ as a soft electrode in our microfluidic chips, the logic architecture was greatly simplified, and the chip size was significantly minimized as a result of micro/nano imprinting techniques on 
AgPDMS conducting composite. While voltage is introduced to help the GERF response to fluids with different dielectric properties, the logic function is carried solely by the fluid- no active electrical control is involved in this process-pure fluid logic is achieved.

\section{Principle and results}

\section{Characteristics of giant electrorheological fluid}

Electrorheological fluid (ERF) ${ }^{18,19}$ is a type of well-known smart material composed of dielectric particles suspended in an insulating oil, forming a two-phase system. As illustrated in Fig. 1(a), the dielectric particles can be polarized under an electrostatic field with an effective dipole moment. Under this induced dipoledipole interaction, the particles tend to aggregate and form columns in the applied field direction. ${ }^{14}$

ERF exhibits solid-like behavior sustaining shear in the direction perpendicular to the applied electric field, and the shear stress can be enhanced when the applied electric field increases. This rheological variation is reversible upon removal of the field. The response time can be as short as a few milliseconds. Due to such marvellous features, ERF can serve as an electric-fluidmechanical interface, responding to electrical signals, providing dynamic force, while working as input signals for electrical devices such as clutches, valves, dampers and others. Hence ERF is denoted a "smart" colloid. However, in spite of ERF's promisingly wide utility and the correspondingly broad interest shown in it, applications have been hampered by the low yield stress of the electrorheological (ER) effect. Considerable efforts have gone into the preparation of alternative suspended particles with the aim of improving the ER yield stress. The discovery of the giant electrorheological (GER) effect, ${ }^{\mathbf{1 4 2 0 - 2 3}}$ however, represented a paradigm that changed conventional ER mechanism. The GER yield stress can be as high as $300 \mathrm{kPa}$ - the strongest ER effect yet tested. ${ }^{24,25}$ The phase change electrical field could reach $2000 \mathrm{~V} \mathrm{~mm}^{-1}$, which is perfectly suitable for manipulation in microfluidic devices in which the typical channel size is 10-200

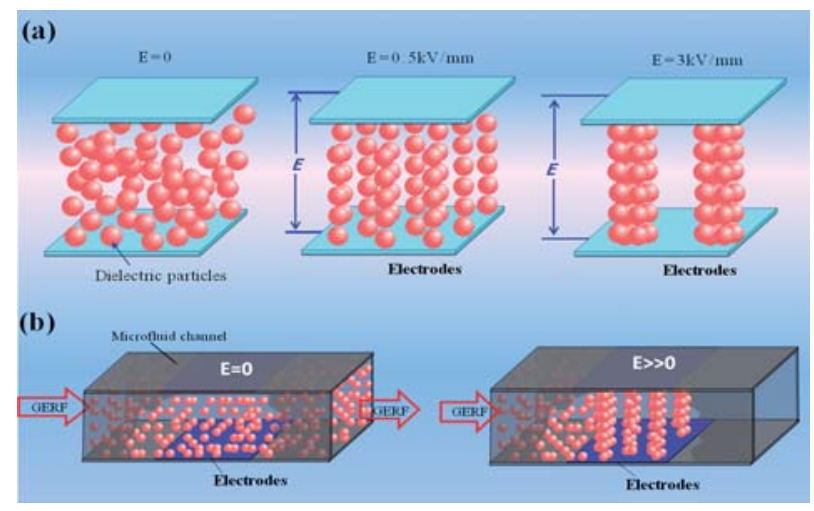

Fig. 1 Working principle of GERF. (a) GERF stays in the liquid state when no electric field is applied, begins to form chains when an electric field is applied, then grows into columns when the electric field increases. (b) When no electric field is exerted, GERF keeps flowing because it is pumped, and when the electric field is applied on GERF by embedded parallel plate electrodes, GERF solidifies. A large enough electric field will provide very high yield stress of GERF, resulting in a fluid suspending effect. $\mu \mathrm{m}$. This discovery facilitated the application of ERF in various devices, especially in GERF-based (dielectric constant $\varepsilon=60-80$ ) microfluidic logic devices for precise fluid and droplet control, from basic functions of microfluidic pumps, valves and mixers, to more advanced logic functions such as droplet encoding/decoding modules, ${ }^{12}$ droplet display and droplet storage systems, etc. The working scheme is illustrated in Fig. 1(b), when there is no electric field applied on GERF, GERF keeps flowing in the microfluidic channel, and when an electric field is applied, shown on the right side of Fig. 1(b), GERF starts to form columns, leading to high yield stress to balance the pumping pressure, and will block the flow if the electric field is strong enough.

The high GER effect is a function of the GER particles - ureacoated barium titanyl oxalate (BTRU) described in our previous work. ${ }^{22}$ In the presence of a saturated urea solution, solutions of barium chloride, titanium tetrachloride, and oxalic acid were coprecipitated at $65{ }^{\circ} \mathrm{C}$ to form colloidal sols. The mixture was washed was de-ionized water to remove aqueous acid. After suction filtration, extra water was removed by vacuum-dryer: the drying time should be well-controlled so that the GER particles have the correct amount of water for strong ER effect and high breakdown voltage. To make the GERF more suitable for the application in our microfluidic chips, dried GER particles were then dispersed in sunflower oil by weight concentration of $40 \%$ and ball milled for 30 minutes for better suspension and distribution of GER particles. To avoid large size clusters blocking the microfluidic channel, the mixture was further filtered by sieve (350 mesh).

\section{Microfluidic chip fabrications}

We purposely choose polydimethylsiloxane (PDMS) as bulk material, because it is an inexpensive silicon rubber which is flexible, ${ }^{26}$ transparent, and totally biocompatible. ${ }^{27}$ Effective manipulation of GERF in PDMS microfluidic chips requires micro-sized parallel plate electrodes for a uniform electric field. However, PDMS doesn't bond with metal, which results in difficulties when inserting metal electrodes or wires. A new group of conductive composites, PDMS-based conductive composites, ${ }^{17}$ were invented to solve this problem. This new group of conductive composites are a mixture of microsized granules of silver powder and PDMS gel. The electrical conductivity of this type of composite increases as the weight percentage of silver powder increases, and can reach as high as $5 \times 10^{4}\left(\mathrm{~S} \mathrm{~m}^{-1}\right)$, while the material maintains good flexibility and processability. The PDMS-based nature ensures its perfect bonding with PDMS chips, and standard photolithographic processes are perfectly applicable to this new type of material. Moreover, microfluid channel wall embedded plate electrodes can be constructed by standard soft lithographic techniques.

The fabrication processing of our chip is illustrated as follows: Firstly, a glass wafer was cleaned in a $70{ }^{\circ} \mathrm{C}$ bath in solution $\left(\mathrm{NH}_{4} \mathrm{OH}: \mathrm{H}_{2} \mathrm{O}_{2}: \mathrm{H}_{2} \mathrm{O}=1: 1: 5\right)$ for $15 \mathrm{~min}$, followed by DI water washing, compressed $\mathrm{N}_{2}$ gas drying and baking in an oven at $120^{\circ} \mathrm{C}$ for over $30 \mathrm{~min}$. Then, photoresist SU-8 2050 was spincoated onto the wafer at $1700 \mathrm{rpm}$.

After soft baking, the wafer was exposed to UV with an energy of $600 \mathrm{~mJ} \mathrm{~cm}{ }^{-2}$, hard baked and developed in SU8 developer to obtain the desired mold pattern for the flow channels, as shown 


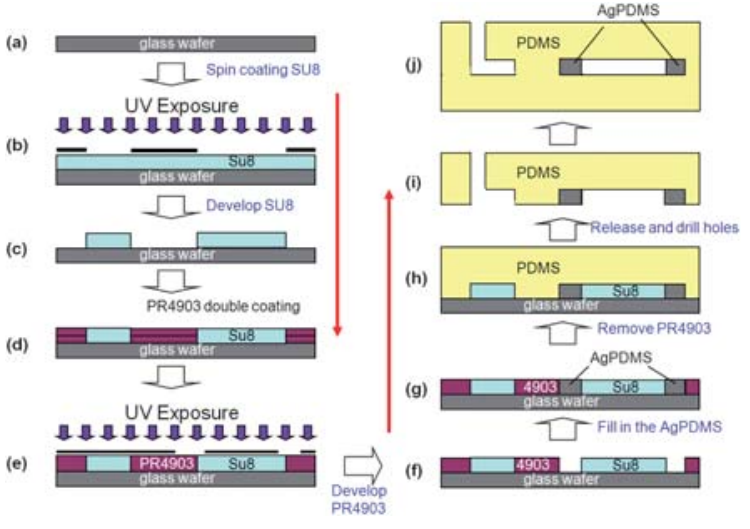

Fig. 2 Schematic view of fabrication process.

in Fig. 2(a)-(c). Another photoresist PR-4903 forms concave patterns for the conducting component. Its fabrication process is demonstrated in Fig. 2(d)-(f). It was double-coated at the spin rate of $800 \mathrm{rpm}$, baked and exposed to UV with an energy of $2000 \mathrm{~mJ} \mathrm{~cm}{ }^{-2}$. After developing in AZ400K : $\mathrm{H}_{2} \mathrm{O}=1: 3$ (volume ratio), the layer of PR4903 had a thickness similar to that of SU8, with the desired pattern. PDMS pre-polymer was prepared by mixing silicone elastomer base and curing agent at a weight ratio of $10: 1$. Silver particles $1-2 \mu \mathrm{m}$ in size were mixed into the PDMS pre-polymer and ground with a mortar for uniform distribution. The mixture was then filled into the cavities of PR4903 as shown in Fig. 2(g). After baking in an oven to fully solidify the Ag-PDMS, PR4903 was removed by bathing in acetone $1 \mathrm{~min}$, ethanol $1 \mathrm{~min}$ and DI water $1 \mathrm{~min}$. The whole device was then put in the oven again to remove the remaining water before the PDMS pre-polymer was poured on it and solidified in a $60{ }^{\circ} \mathrm{C}$ oven for about $2 \mathrm{~h}$ (Fig. 2(h)). The solidified PDMS slab was taken off the substrate with the fluid channel structures and Ag-PDMS pattern embedded, and holes for outlets were drilled, as shown in Fig. 2(i). Finally, this PDMS slab was bonded with another flat PDMS piece with half-cured bonding, and the microfluidic chip with embedded electrodes for our logic gate was obtained in Fig. 2(j).

The whole device is sketched in Fig. 3(a). The cyan lines indicate the fluid channels and the purple lines are the conducting component. Most of the fluid channels were about $200 \mu \mathrm{m}$ wide and $100 \mu \mathrm{m}$ in depth. Fluids were pumped into the chip by syringe pump (kdScientific). Signal droplets were generated by a T-junction structure and output GERF droplets are formed at

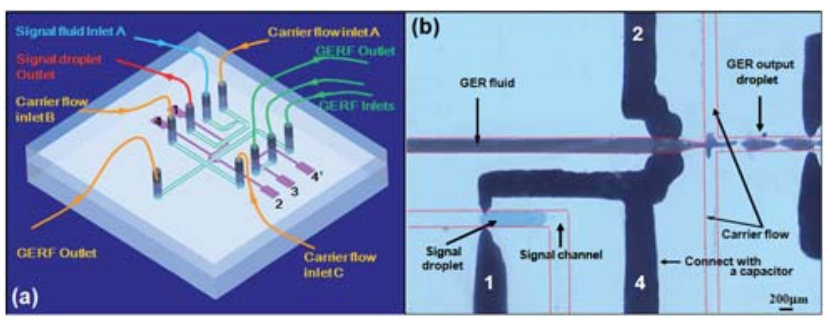

Fig. 3 PDMS-based chip for microfluidic logic control. (a) Schematic view of the chip design for the microfluidic switch/inverter. Both functions can be realized in this chip by rearranging the voltage input. (b) Optical picture (enlarged view) of the key part where the logic operation takes place. the flow focusing part. The AgPDMS-fabricated signal electrodes had a width of $50 \mu \mathrm{m}$, and the responsive output electrodes were $400 \mu \mathrm{m}$ in width in order to effectively stop the GERF. Five electrode pads were designed for external connection, while three pairs of electrodes were located on the sides of channels, two pairs of them detecting the signal droplets and one for controlling the GERF. Electrode pads 1, 2, and 3 are for DC voltage supply to define logic operations, and electrodes 4 and $4^{\prime}$ are connected by external wire to electrically connect the fluidic capacitor $\left(\mathrm{C}_{3}\right)$ to the fluidic channels for setting the switch function (IF gate). The microfluidic chips were monitored by a CCD (SONY DXC-390P) installed on a microscope (OLYMPUS SZX16). Pictures and videos were taken using software on a connected computer.

A real image showing a closer view of the logic operation component is shown in Fig. 3(b). The cyan droplet is the signal fluid, carried by silicon oil. The darker GERF fluid is also carried by silicon oil downstream of the flow focusing spot. The electrodes connecting the power supply and fluidic channels are marked with the numbers 1, 2 and 4, which are consistent with the numbers shown in Fig. 3(a).

\section{Logic functions demonstration}

Our basic idea is to use fluid channels with embedded parallel electrodes to replace some of the impedances in an electric circuit. As droplets and carrier fluid could have different dielectric constants or conductivities, a fluid channel can provide variable impedance. When constant voltage is applied, the output will change according to droplet/carrier-fluid alternation between the electrodes embedded on the sides of the fluid channel. In our settings, the output voltage between electrodes 2 and 4 applies to the GERF channel to control the GER droplet generation. Thus the generated GER droplet sequence will represent the output voltage signal, which is decided by the arrangement of the circuits and the variable impedances caused by the droplet input. Moreover, most parts of this micro-circuit could be directly built onto the microfluidic chip by soft lithography techniques and using AgPDMS conducting wires.

Here we demonstrate two basic logic functions, a switcher (IF gate) and invertor (NOT gate). Input signal was defined as binary 1 when an ionized/high dielectric droplet fills the space between electrodes (signal electrodes), and binary 0 when there is only carrier fluid (insulating/low dielectric fluid). Similarly, the output signal was defined as binary 1 when GERF flows out of the flowfocusing spot, and binary 0 when GERF is stopped and carrier fluid flows out.

For example, the microfluid inverter is illustrated in Fig. 4. To achieve this function, signal electrodes and output electrodes (electrodes on the sides of the GERF channel) were in series connection, while the input voltage is applied across electrodes 1 and 2. In this case, the signal fluid is an ionized, high-conductivity solution, which can be modeled as a resistor or conductor, and the carrier flow is an insulating fluid. When the carrier flow presents between the signal electrodes, the circuit could be viewed as open, and the GERF continues flowing (output =1); when the signal droplet is present between the signal electrodes, as it is conductive, the circuit could be viewed as closed, thus voltage is applied to the output electrodes and stops the flow of the GERF 


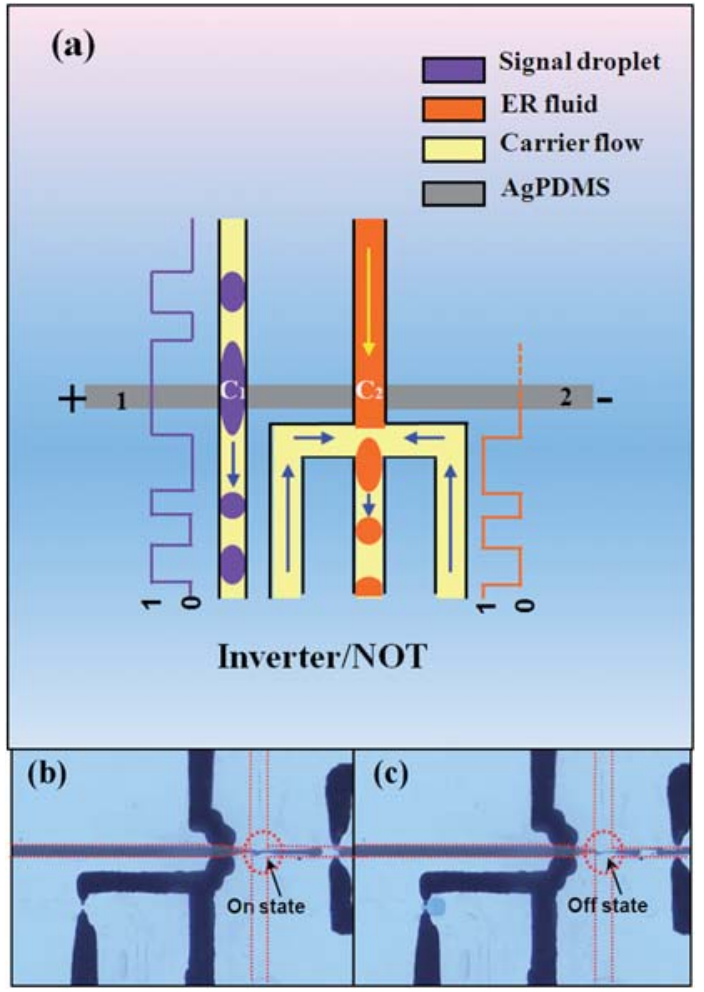

Fig. 4 Basic working principle of a logic gate, illustrated by a microfluid inverter. (a) Schematic of the basic working principle. The presence of a signal droplet between the signal electrodes will solidify GERF, while the presence of carrier flow will release GERF. (b) When the carrier flow is flowing between the signal electrodes, GERF flows continuously. (c) When the signal droplet passes by the signal electrodes, GERF solidifies between electrodes, and is cut into droplets.

(output $=0$ ). The input voltage is set to a value that guarantees solidification of GERF when the signal droplet is present and should be less than that crucial value when the carrier flow passes by the signal electrodes.

This setting also works for the dielectric signal droplet which has a different dielectric constant to the carrier fluid. Then, the fluid in the signal channel can be modeled as a capacitor, with capacitance denoted $C_{1}(x), x=0$ if the input is carrier flow, and $x=1$ if the input is signal droplets, while the capacitance generated by the GERF is denoted as $C_{2}$. In this case, if the applied voltage is $V$, and voltage share of the GERF is $V_{E R}(x)$, we can obtain

$$
V_{E R}(x)=\frac{C_{1}(x)}{C_{1}(x)+C_{2}} V
$$

and we can always adjust the input voltage $V$ to ensure that $V_{E R}(1)$ is larger than the GERF solidification voltage and $V_{E R}(0)$ is smaller than the crucial value. By applying this principle, a fluidic inverter (NOT gate) can be realized.

In our experiment, we choose signal fluid to be a saturated $\mathrm{KOH}(\mathrm{BDH})$ solution $\left(22^{\circ} \mathrm{C}\right)$ dyed blue, which is pumped at a rate of $0.01-0.04 \mathrm{ml} \mathrm{h}^{-1}$, and the carrier flow to be silicon oil (CLEARCO), pumped at flow rate of $0.01-0.05 \mathrm{ml} \mathrm{h}^{-1}$. GERF was injected with a flow rate of $0.01-0.04 \mathrm{ml} \mathrm{h}^{-1}$, while its carrier flow (silicon oil) was pumped at $0.01-0.05 \mathrm{ml} \mathrm{h}^{-1}$. We applied 200 $\mathrm{V}$ to electrode 1 , and $-200 \mathrm{~V}$ to electrode 2 , to meet the phase changing requirement of GERF. As shown in Fig. 4(b), when the fluid that passes the signal electrodes is silicon oil (input $=0$ ), a low voltage is shared on the electrodes located at the GERF channel, GERF keeps flowing (output $=1$ ); while GERF stops flowing (output $=0$ ) as a signal droplet presents between the signal electrodes (input $=1$ ), shown in Fig. 4(c). An inverter is also a fluidic NOT gate, the binary truth table of which is shown in Table 1.

A fluidic switch is designed according to a similar principle as the logic inverter, but the logic state is reversed by electrically connecting a capacitor, as shown in Fig. 5(a). The capacitor should have a capacitance comparable with GERF, in order to effectively share and reduce the voltage applied to the GERF output channel; when the signal droplet is present between the signal electrodes, the voltage share of the GERF is smaller than the crucial value for its solidification. A simple design methodology is to set the voltage input on electrode 1 to be the inverse voltage of the one input on electrode 2, as shown in Fig. 5(a). When the signal droplet is dielectric fluid, we can derive a simplified capacitance model: the input voltage from electrodes $1,2,3$ is $V_{1}, V_{2}$ and $V_{3}$, the capacitances in the signal channel and GERF between the output electrodes have the same definition as in the former inverter configuration, and $C_{3}$ is the capacitance of the added capacitor. $V_{E R}(x)$, the voltage share of the GERF under a different input situation, can be calculated as:

$$
V_{E R}(x)=\frac{\left(V_{2}-V_{1}\right) C_{1}(x)+\left(V_{2}-V_{3}\right) C_{3}}{C_{1}(x)+C_{2}+C_{3}}
$$

where $V_{1}, V_{2}$, and $V_{3}$ can be tuned for a desired voltage arrangement making the GERF solidify when a signal droplet comes by, and change back to the fluid state when the droplet passes the signal electrodes. To rule out variation of the dielectric constant between different batches of GER powder, we used an additional steady GERF channel with embedded electrodes, of the same dimensions as the channel and electrodes in the GERF output channel, as the additional capacitor (shown in Fig. 3(a)). Meanwhile, the case of ionized buffer signal droplets is straightforward to understand, which can be illustrated by our experimental result.

A logic switch was constructed, as shown in Fig. 5(a). Electrodes 1,2 and 3 were set to be $V_{1}=V_{2}=-400 \mathrm{~V}$, and $V_{3}=400$ $\mathrm{V}$, while electrode 4 and $4^{\prime}$ are electrically connected by an external metal wire. The experimental fluids are the same as those tested in the inverter case. Fig. 5(b) and 5(c) show the sequential logic response of the switch. When the input droplet signal was false (input $=0$ ), as shown in Fig. $5(\mathrm{~b}), V_{E R}(0)=400 \mathrm{~V}$, resulting in an electrical field $E_{E R}(0)=2000 \mathrm{~V} \mathrm{~mm}^{-1}$, and the GERF was solidified (output $=0$ ); when the input droplet signal was true (input $=1$ ), as shown in Fig. 5(c), $V_{E R}(1)=0 \mathrm{~V}$, and no electric field was applied to the ERF output channel (output $=0$ ). This fluid switch can also function as a logic IF gate, the truth table of which is shown in Table 1 .

\section{Discussions}

The advantage of our microfluidic logic switch and inverter is that two counterpart functions can be realized using one identical simple chip structure. Alternation between the two functions is achieved by selective voltage inputs, or by connecting alternative 
Table 1 Working state and truth table of microfluidic logic switch and inverter. Output signal is demonstrated by GERF flowing status. Continuing or resumed flow represents binary "1", while suspended GERF means binary "0".

Truth table for microfluidic logic control device

\begin{tabular}{|c|c|c|c|c|}
\hline & Input droplet & $\begin{array}{l}\text { Input } \\
\text { Signal }\end{array}$ & GERF state & $\begin{array}{l}\text { Output } \\
\text { signal }\end{array}$ \\
\hline Inverter & Carrier flow & 0 & flowing & 1 \\
\hline (NOT gate) & Signal droplet & 1 & suspended & 0 \\
\hline Switch & Carrier flow & 0 & suspended & 0 \\
\hline (IF gate) & Signal droplet & 1 & flowing & 1 \\
\hline
\end{tabular}

electrode pads. As the logic switch and inverter are the basic units for logic operations, a system allowing this logic unit can have more functions just by rearranging the voltage input, without any other reconstruction of physical structures.

The complete output droplet always forms downstream of the flow focusing spot. The phase changing response of GERF to signal droplets is within $10 \mathrm{~ms}$, which is almost instantaneous. However, output information carried by GERF droplets incorporates the fluid cutting effect of the carrier flow. The flowfocusing structure is the spot where the output droplet starts to form, and it always takes $50-200 \mathrm{~ms}$ to complete this process. The actual formation time depends on the flow rate of both the

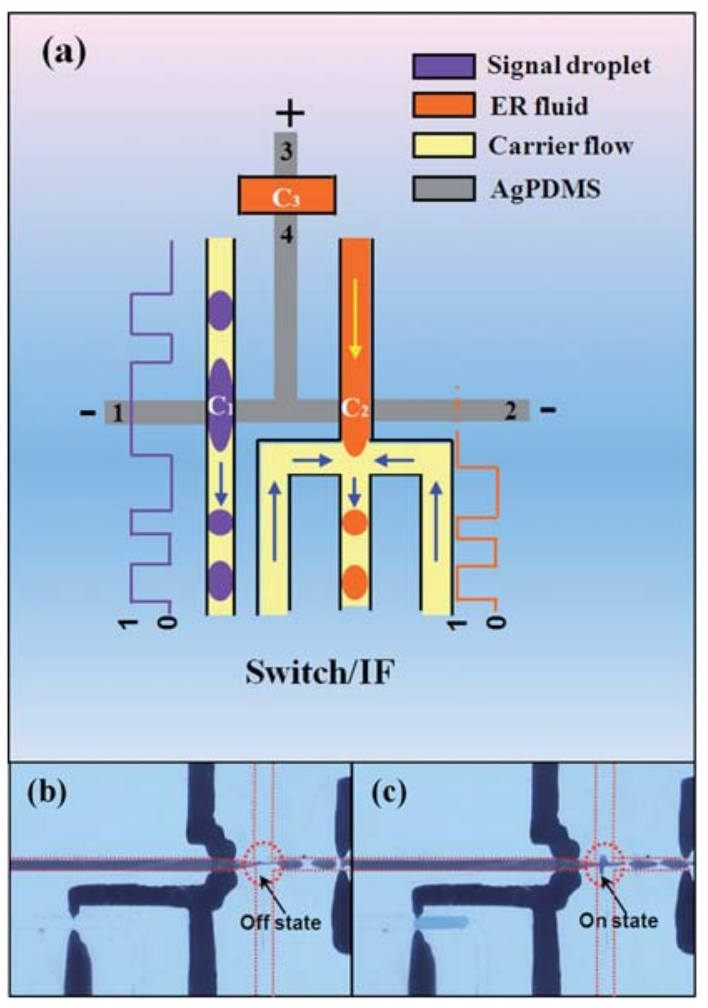

Fig. 5 Working principle for a microfluidic switch. (a) A microfluid logic switch is build by adding a capacitor (another microfluid channel filled with steady GERF in our case) to the logic inverter. (b) When carrier flow presents between the signal electrodes, GERF solidifies. (c) When signal droplets present between the signal electrodes, GERF liquefies and flows out.
GERF and associated carrier flow. On-going chemical modification research on GER powder continues for a faster response, and the flow-focusing time can also be varied by tuning the flow rates of both GERF and its carry flow: these two modifications may greatly reduce the droplet formation time. This time delay does not severely affect the logic process, because our logic chip doesn't mean to compete with traditional electronics in speed: we deal with droplets in which cells could be cultured or chemical droplets could be individually addressed. In a further integrated system, a droplet-phase module could be added by established techniques. ${ }^{13}$

The choice for the signal droplets and carrier flow is very flexible, as long as there exits a dielectric property difference between the two fluids. Therefore, given any kind of signal fluid, we can chose an appropriate carrier flow to form an effective logic operation. For example, in our logic inverter, we can reassign silicon oil as signal droplets, and $\mathrm{KOH}$ solution as carrier flow, then it works as a switch for an input signal of silicon oil. The truth table for which is shown in Table 2.

Moreover, effective assembly of the proposed switch and inverter might realize additional microfluidic logic functions. The prominent GERF property of being a fluid-mechnical-electronic interface suggests further external electronic/internal fluidic computing possibilities after one operation, and ultimately large scale integration for multipurpose biochemical/chemical information processing. This specific logic module can be integrated with our previous GERF based fluid logic control modules, such as the droplet phase exchange module, ${ }^{13}$ to realize droplet synchronization, or other advanced logic operations. Possible applications include multi-component serial reactions or automatic reagent loading for multi-step biological/chemical operation, such as the investigation of time and quantity control in drug release.

\section{Conclusions}

We report a microfluidic logic switch and inverter realized in a single microfluidic chip structure where GERF is generated as the output signal. We used a voltage supply to realize the computing function, while the logic process was carried out solely by the droplet signal inputs. Application of a AgPDMS conducting composite to this logic gate enables the logic module to be integrated with electronic monitoring and other electronic equipment. The output signal was a GERF droplet, which could be stored in the microfluidic display ${ }^{12}$ system or utilized as an input signal to control subsequent information processing. ${ }^{15}$ The design of the suggested chip is a simple single-layer fluidic

Table 2 Working state and truth table of a microfluidic logic switch, when the signal droplet is set to be an insulating fluid, or fluid with low dielectric constant

Truth table for microfluidic switch (reverse definition of signal droplet)

\begin{tabular}{lllll}
\hline & $\begin{array}{l}\text { Input } \\
\text { droplet }\end{array}$ & $\begin{array}{l}\text { Input } \\
\text { Signal }\end{array}$ & $\begin{array}{l}\text { ERF } \\
\text { state }\end{array}$ & $\begin{array}{l}\text { Output } \\
\text { signal }\end{array}$ \\
\hline $\begin{array}{rlll}\text { Switch (IF gate for } \\
\text { silicon oil signal) }\end{array}$ & $\begin{array}{l}\text { Silicon oil } \\
\text { KOH buffer }\end{array}$ & $\begin{array}{l}1 \\
\text { flowing }\end{array}$ & $\begin{array}{l}1 \\
\text { suspend }\end{array}$ & 0 \\
\hline
\end{tabular}


structure, which is easy to realize by standard soft lithographic techniques, with which large-scale integration is easily achieved.

\section{Acknowledgements}

This publication is based on work partially supported by Award No. SA-C0040/UK-C0016, made by King Abdullah University of Science and Technology (KAUST), Hong Kong RGC grants HKUST 603608, and the Nanoscience and Nanotechnology Program at HKUST. Useful discussion with Casey Glick is acknowledged.

\section{Notes and references}

1 R. B. Fair, Microfluid. Nanofluid., 2007, 3, 245-281.

2 M. Abdelgawad and A. R. Wheeler, Adv. Mater., 2009, 21, 920-925.

3 V. Srinivasan, V. K. Pamula and R. B. Fair, Lab Chip, 2004, 4, 310-315.

4 M. Prakash and N. Gershenfeld, Science, 2007, 315, 832-835.

5 T. Vestad, D. W. M. Marr and T. Munakata, Appl. Phys. Lett., 2004, 84, 5074-5075.

6 A. Groisman, M. Enzelberger and S. R. Quake, Science, 2003, 300, 955-958.

7 J. A. Weaver, J. Melin, D. Stark, S. R. Quake and M. A. Horowitz, Nat. Phys., 2010, 6, 218-223.

8 B. Lin, Y. Gao and J. Qin, J. Chin. Chem. Soc., 2009, 56, 1-15.

9 G. M. Whitesides, Nature, 2006, 442, 368-373.

10 X. Niu, W. Wen and Y. Lee, Appl. Phys. Lett., 2005, 87, 243501.
11 L. Liu, X. Chen, X. Niu, W. Wen and P. Sheng, Appl. Phys. Lett., 2006, 89, 083505 .

12 X. Niu, M. Zhang, J. Wu, W. Wen and P. Sheng, Soft Matter, 2009, 5, $576-581$

13 M. Zhang, J. Wu, X. Niu, W. Wen and P. Sheng, Phys. Rev. E, 2008, 78, 066305.

14 W. Wen, X. Huang and P. Sheng, Soft Matter, 2008, 4, 200.

15 X. Niu, M. Zhang, S. Peng, W. Wen and P. Sheng, Biomicrofluidics, 2007, 1, 044101.

16 P. S. Dittrich, Lab Chip, 2009, 9, 1165-1166.

17 X. Niu, S. Peng, L. Liu, W. Wen and P. Sheng, Adv. Mater., 2007, 19, 2682-2686.

18 R. Tao and J. M. Sun, Phys. Rev. Lett., 1991, 67, 398-401.

19 D. J. Klingenberg, F. Van Swol and C. F. Zukoski, J. Chem. Phys, 1989, 91, 7888-7895.

20 H. Ma, W. Wen, W. Y. Tam and P. Sheng, Adv. Phys., 2003, 52, $343-$ 383.

21 W. Y. Tam, G. H. Yi, W. Wen, H. Ma, M. M. T. Loy and P. Sheng, Phys. Rev. Lett., 1997, 78, 2987-2990.

22 W. Wen, X. Huang, S. Yang, K. Lu and P. Sheng, Nat. Mater., 2003, $2,727-730$.

23 W. Wen, X. Huang and P. Sheng, Appl. Phys. Lett., 2004, 85, 299301.

24 C. Shen, W. Wen, S. Yang and P. Sheng, J. Appl. Phys., 2006, 99, 106104.

25 L. Liu, X. Huang, C. Shen, Z. Liu, J. Shi, W. Wen and P. Sheng, Appl. Phys. Lett., 2005, 87, 104106.

26 J. C. McDonald, D. C. Duffy, J. R. Anderson, D. T. Chiu, H. Wu, O. J. A. Schueller and G. M. Whitesides, Electrophoresis, 2000, 21, $27-40$.

27 S. K. Sia and G. M. Whitesides, Electrophoresis, 2003, 24, 3563-3576. 